Tamar Katzav-Gozansky · Raphael Boulay •

Robert Vander Meer · Abraham Hefetz

\title{
In-nest environment modulates nestmate recognition in the ant Camponotus fellah
}

\begin{abstract}
Multiple behavioral and chemical studies indicate that ant nestmate recognition cues are low-volatile substances, in particular hydrocarbons (HCs) located on the cuticular surface. We tested the hypothesis that in the ant Camponotus fellah, nest environment, in particular nest volatile odors, can modulate nestmate-recognitionmediated aggression. Workers were individually confined within their own nest in small cages having either a single mesh (SM = limited physical contact permitted) or a double mesh $(\mathrm{DM}=$ exposed to nest volatiles only) screen. Individual workers completely isolated outside their nest (CI) served as control. When reintroduced into a group of 50 nestmates, the CI workers were attacked as alien ants after only 2 weeks of separation, whereas the SM workers were treated as nestmates even after 2 months of separation. Aggression towards DM ants depended on the period of isolation. Only DM workers isolated for over 2 months were aggressed by their nestmates, which did not significantly differ from the CI nestmates. Cuticular $\mathrm{HC}$ analyses revealed that the profile of the nonisolated ants (NI) was clearly distinct from that of CI, SM and DM ants. Profile differences matched the aggressive response in the case of $\mathrm{CI}$ ants but were uncorrelated in the case of SM or DM ants. This suggests that keeping the ants within the nest environment affected nestmate recognition in additional ways than merely altering their HC profile. Nest environment thus appears to affect label-template mismatch by modulating aggressive be-
\end{abstract}

\author{
T. Katzav-Gozansky () ) A A. Hefetz \\ Department of Zoology, George S. Wise Faculty of Life Sciences, \\ Tel Aviv University, Ramat Aviv, Tel Aviv, Israel \\ e-mail: katzavt@post.tau.ac.il \\ Tel.: +972-3-6408766 \\ Fax: $+972-3-6406991$ \\ R. Boulay \\ Estación Biológica de Doñana, \\ CSIC, Apdo 1056, 41013 Seville, Spain \\ R. Vander Meer \\ USDA/ARS, CMAVE 1600 SW 23 Drive, Gainesville, \\ FL, 32608 USA
}

havior, as well as the direction at which cuticular HCs diverged during the separation period.

\section{Introduction}

Nestmate recognition determines, to a large extent, the outcome of interactions between encountering ants. Recognizing the encountered ant as alien provokes high aggression, as opposed to nestmates that elicit amicable interactions such as trophallaxis. In ants, it is widely accepted that low-volatile chemicals that reside on the cuticle, among which cuticular HCs appear prominent, are the basis discriminators (Thomas et al. 1999; Lahav et al. 1999; Wagner et al. 2000). The use of low-volatile compounds as nestmate recognition cues seems to be adaptive by limiting the response to close range and upon contact with the encountering ant. However, studies in ants as well as honeybees suggest that additional, more volatile, substances may also be involved in the process (Jaffe and Marcuse 1983 for ants; Mann and Breed 1997 for honeybees). Furthermore, behavioral studies in social halictine bees (Lasioglossum) (Kukuk et al. 1977) and the social wasp Polistes fuscatus (Gamboa et al. 1986) have suggested that nest odors are largely involved in nestmate recognition. While nest environment can affect the composition of the recognition cues of nest members, it may also act indirectly by affecting the manifestation of nestmate recognition through influencing worker behavior and physiology (Boulay et al. 2003a). Earlier studies with C. fellah have shown that short isolation period of ants from their mother colony results in small HC profile changes and promotes elevated trophallaxis, presumably to conform to the original colony profile (Boulay et al. 1999). Longer isolation periods, on the other hand, resulted in major HC profile shifts that were accompanied by high aggression (Boulay et al. 2003b). These findings, however, may be confounded by the fact that total isolation may affect the ants in more ways than just preventing them from conforming to the colony odor. For example, the in-nest environment, including odors ema- 

nating from nest substrate, food, feces, the queen and brood may modulate the ant aggressive and or amicable responses.

The aim of the present study was to investigate the possible impact of nest environment on the nestmate recognition process in C. fellah, through the perception of volatile compounds emitted by colony members. We studied the effect of depriving workers of contact and/or volatile cues present in the nest on nestmate recognition, by confining individual ants in SM or DM cages within their own nest.

\section{Method}

Animals

Colonies of C. fellah were established and reared as previously described (Boulay at al. 2003a). To neutralize a possible effect of the diet on HC composition (Liang and Silverman 2000), the ants were fed during the experiment with sugar water only. Colonies selected for the experiments had attained the size of at least 500 workers (total of eight colonies).

Isolation of individual ants within their colony

Workers (50 in each nest) were isolated within their nest in SM or DM cages. A third set of cages contained CI workers. The cages comprised Petri dishes (3.5 cm diameter), the covers of which were replaced by single (9-mesh screen) or double wire mesh (50-mesh screen). Thus, SM workers had limited physical contact with their nestmates, while DM workers could only exchange volatile cues. The isolated ants were kept in the colony for up to 63 days, with weekly samples being removed for bioassay.

\section{Behavioral assays}

Dyadic encounters for measuring trophallaxis

Dyadic encounters were modified from the bioassay used by Boulay et al. (1999) and were aimed at scoring amicable interactions after 10 days of isolation. Trophallaxis was used for assessing nestmate recognition, since it was found to be a sensitive aspect of recognition (Boulay et al. 2003b). Two workers, resident and confined, were marked with a dot of paint on their thorax and placed in two different test tubes $(7.5 \times 0.9 \mathrm{~cm})$ for acclimation. At the onset of the assay, the test tubes were gently connected. CI, SM, and DM workers were confronted for 10 min with NI nestmates (3-4 ants per colony per treatment). Control encounters were conducted between two NI nestmates. Behaviors were continuously recorded for $10 \mathrm{~min}$ from the first contact between the two ants.

Group encounter bioassays for measuring aggression

Bioassays for measuring the tolerance of the colony towards the confined nestmates were performed in a circular arena $(9 \times 7 \mathrm{~cm})$ containing 50 resident workers into which a marked ant was introduced (2-4 ants per colony per treatment). The resident workers were placed in the arena for 30-60 min before each test to allow acclimation. Two observation-sessions were conducted, 3 and 40 min after presenting the introduced ant. The aggressive behavior (threats with open mandibles, bites, and gaster flexion) performed by the resident ants toward the introduced ant was recorded 20 times, every $10 \mathrm{~s}$. For statistical analysis, the total numbers of aggressive interactions were averaged between the two trials.
Chemical analysis

For chemical analysis, the postpharyngeal glands (PPG) of 21-day isolated ants were extracted in $50 \mathrm{ml}$ of pentane containing $1 \mathrm{mg}$ of heptadecane as internal standard. Cuticular HCs were obtained by immersing each thorax for $10 \mathrm{~min}$ in $200 \mathrm{ml}$ of pentane containing $2 \mathrm{mg}$ of heptadecane. Analysis of PPG and cuticular HCs were done by gas chromatography (Varian CP 3800) equipped with DB-1 fused silica column temperature programmed from $150-300^{\circ} \mathrm{C}$ at $5^{\circ} \mathrm{C} / \mathrm{min}$. Cuticular HCs and PPG lipids identification by GC/MS was based on Boulay et al. (2003a). Only peaks that were reliably and reproducibly quantifiable were utilized for the statistical analyses.

\section{Statistical analysis}

Two-way ANOVA was used to compare durations of trophallaxis in dyadic encounters. Worker treatment was introduced in the model as a fixed factor with four levels (CI, DM, SM, and NI) while the colony of origin was introduced as a random factor with eight levels. For within-group encounters, the frequency of aggressive items was compared by two-way ANOVA (time after separation crossed with treatment of the introduced ant). When the ANOVA was significant, LSD post-hoc tests were used to check between-groups differences. Cuticular and PPG HC profiles were compared using forward stepwise discriminant function analyses.

\section{Results}

\section{Dyadic encounters}

Total duration of trophallaxis between the two ants after 10 days of isolation was affected by the treatment, e.g., type of separation, but not by the colony (Fig. 1; two-way ANOVA: $\mathrm{F}_{3,21}=4.12, \mathrm{P}=0.02$ and $\mathrm{F}_{7,83}=1.01, \mathrm{P}=0.43$, respectively). Trophallaxis duration between NI nestmates (control) averaged (€SE) 74.7€20.5 s, representing a basal level under our bioassay conditions. In contrast, trophallaxis duration between NI and CI ants was highly elevated (221.7€27.8 s) and significantly different from the control (LSD post-hoc test, $\mathrm{P}<0.001$ ). Although trophallaxis with

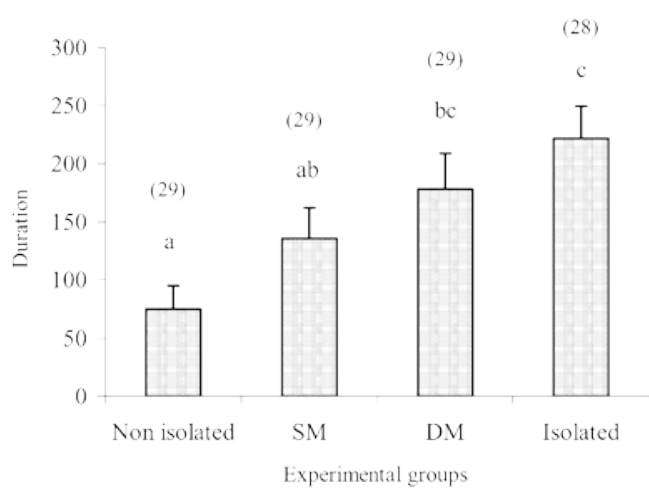

Fig. 1 Mean duration of trophallaxis (€SEM) between non-isolated (NI) workers and workers that were either completely isolated (CI), or isolated by a single-mesh cage (SM) or a double-mesh cage (DM), or also NI. Statistical analysis was performed using ANOVA. Different letters denote statistical differences at $\mathrm{P}=0.05$ according to LSD post-hoc test ( $\mathrm{n}=$ number of replicates) 


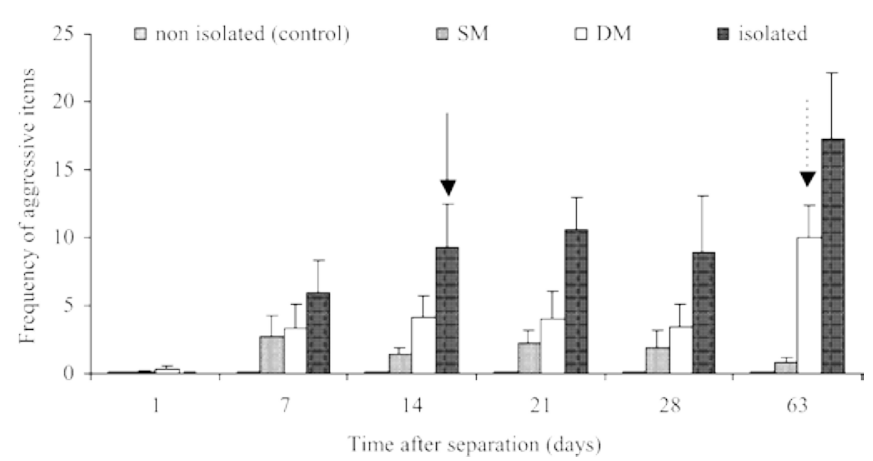

Fig. 2 Mean frequency (€SE) of aggressive behaviors of 50 resident workers towards an introduced nestmate (CI completely isolated, SM isolated with a single mesh, DM isolated with a double mesh, or NI non-isolated)

SM ants was longer than between NI ants, amounting to $135 € 26 \mathrm{~s}$, this difference was not significant (LSD posthoc test, $\mathrm{P}=0.12$ ). Trophallaxis involving $\mathrm{DM}$ ants showed intermediate values (178€31 s) that were not significantly different from either SM or CI ants (LSD post-hoc test, $\mathrm{P}=0.27$ and $\mathrm{P}=0.26$, respectively). It was, however, significantly different from trophallaxis between NI control ants $(\mathrm{P}=0.008)$.

\section{Group encounter bioassays}

The number of aggressive acts performed by resident ants toward the intruders was significantly affected by the time of separation, the treatment of the introduced ant, and their interaction (Fig. 2; two-way ANOVA: $\mathrm{F}_{5,263}=5.37, \quad \mathrm{P}=0.0001 ; \quad \mathrm{F}_{3,263}=23.7, \quad \mathrm{P}<0.0001$, and $\mathrm{F}_{15,263}=2.12, \mathrm{P}<0.009$, respectively). Aggression towards SM intruders was low irrespective of time of separation. Although it was consistently higher than that towards NI ants, this was not significant. By contrast, aggression towards the DM intruders was significantly time-dependent: for the first 28 days post separation, aggressive behavior was still relatively low, not exceeding $4.13 € 1.55$ events per encounter. This was slightly higher than toward SM ants, but was not significantly different from aggression exhibited toward either NI or SM ants. However, after 63 days of isolation, resident workers became less tolerant, reaching an average of 10.0€2.4 aggressive events (dashed arrow in Fig. 2; LSD post-hoc test, $\mathrm{P}=0.03$ with respect to NI ants). Development of aggressive behavior toward CI intruders was rapid and intensive, reaching significant levels on day 14, as compared with control intruders (full arrow in Fig. 2; LSD post-hoc test, $\mathrm{P}=0.004$ ). The aggression towards the CI ants further increased with time, reaching 17.3€4.9 events on day 63 .

\section{HC profiles}

We assessed the possible shift in cuticular HCs as a function of treatment by discriminant function analyses.
A. Wilks' lambda $=0.022 \quad \mathrm{~F}_{48,119}=6.5 \quad \mathrm{P}<0.0001$

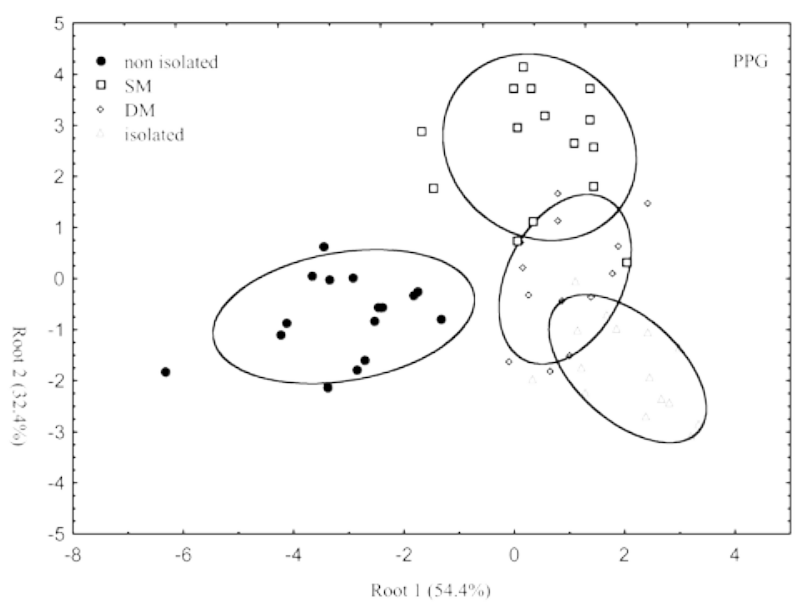

B. Wilks' lambda $=0.042 \quad \mathrm{~F}_{33,142}=8.33 \quad \mathrm{P}<0.0001$

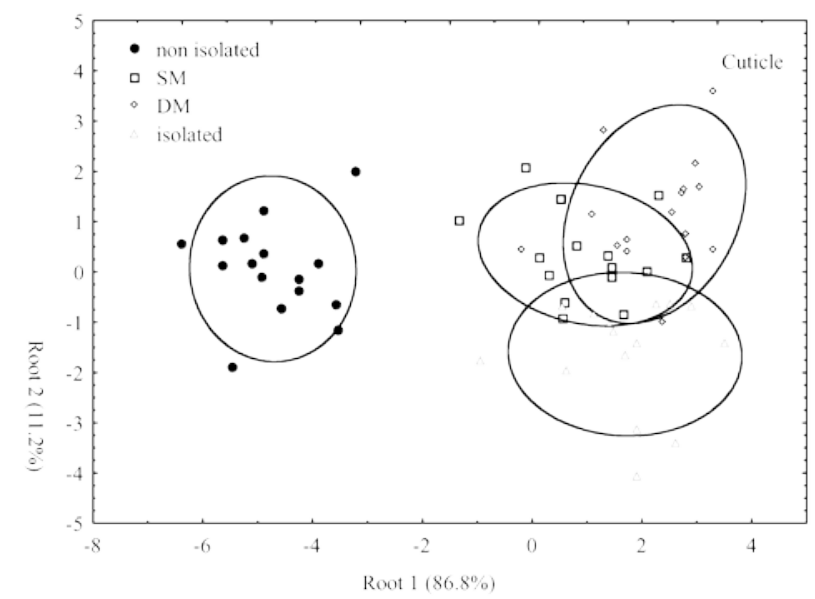

Fig. 3 Discriminant analysis of major hydrocarbon (HC) peaks, 25 for PPG (postpharyngeal glands) (A) and 16 for cuticle (B), of nonisolated (NI) workers, single- (SM) and double-mesh (DM) caged workers, and completely isolated (CI) workers. The ellipses represent the range of distribution under the $95 \%$ confidence interval

Figure 3 depicts the scatter plots for one of the colonies tested. NI ants possessed a profile that was distinct from all the other ant groups, both for the PPG and the cuticle. The PPG HC profile for the CI ants was clearly different from that of the SM ants, while that of the DM showed intermediate profile-similarity, with some overlap with the SM and the CI workers. Separation between the treatment groups using cuticular profiles was less clear. The CI ants had little overlap with the DM ants, whereas the profiles of the SM ants overlapped with both the isolated and the DM ants. 


\section{Discussion}

The prevailing hypothesis regarding nestmate recognition is that a mismatch between label and template of the encountering ants elicits aggression (Vander Meer and Morel 1998). This reaction is not context-independent and may be affected by factors other than the recognition labels. Studies with C. fellah have indicated that the social environment, e.g., the queen or nestmate workers, may have an impact on the process (Boulay et al. 2003a). Although it has been long postulated that nest environment can also affect nestmate recognition, this has not previously been explicitly tested. The different mode of isolation employed in this study enabled a first glimpse at such an effect. We successfully created graded states of social deprivation without completely detaching the ants from the nest environment, in contrast with the CI ants. The results pertaining to the SM ants were as predicted; they were treated as nestmates by the resident ants even after 63 days of isolation. Although aggression was consistently higher than the NI control, it was not statistically different. This shows that even limited physical contact with nestmates is sufficient to maintain their status as nestmates.

Ants separated by a DM were deprived of physical contact with nestmates but were still exposed to general nest odors, resulting in a more complex interaction. Although these workers were eventually aggressed as predicted, alienation was delayed for up to 2 months, suggesting a temporary effect of nest volatiles on recognition. In all cases we verified that aggression was not caused by worker reproduction attempts.

The assessment of whether nest volatiles affect HC profiles gave more equivocal results. Our hypothesis was that SM ants could exchange cues with nestmates and thus conform to the colony profile (Soroker et al. 1994; Meskali et al. 1995), while the profile of the CI ants should diverge the most (Boulay et al. 2003a). We further hypothesized that if nest volatiles affect HC composition, the HC profiles of the DM ants should diverge less than those of the CI ants but more than the SM ants. The analysis revealed a pattern of grouping according to treatments. The dissimilarity in profiles between the SM and NI ants showed that the limited contact possibility was not enough and that there was little exchange of HCs between the SM ants and their nestmates. Furthermore, the profile of the DM ants also diverged from that of the NI ants, suggesting that nest volatiles have little effect on either the PPG or cuticular HC composition. The fact that all the groups of isolated ants were segregated suggests that changes in profiles were independent and non-directional, similar to findings in Cataglyphis niger (Lahav et al. 2001).

Assuming that cuticular HCs provide a reliable model for the dynamics of recognition cues in C. fellah, the differences between the chemical and behavioral data suggest that additional factors are involved. The clear profile differences between the NI and totally isolated ants matched the high aggressive behavior between these two groups. However, aggression between the NI and either the SM or DM ants was lower than predicted from profile differences. This suggests that ants' isolation within the nest environment affected nestmate recognition in additional ways than just altering their HC profile. The fact that aggression towards the SM ants was very low but still consistently higher than the NI control may hint at an interplay between the alienating effects of profile segregation and the familiarity effect of nest volatiles. In the DM cases, aggression was even higher but not significant until day 63, corroborating the interplay hypothesis. We propose that up to 2 months of separation, profile dissimilarity was successfully counteracted by nest-odor familiarity (Errard and Hefetz 1997). After a longer isolation period, profile divergence became sufficiently great to completely alienate the isolated ants. Nest volatiles may also have had a modulatory effect on the SM and DM workers. Since they did not experience any changes in their environment during the isolation period they did not behave irregularly when encountering the NI ants. This, in turn, may have had a pacifying effect on the latter, resulting in high rates of acceptance (Roulston et al 2003).

Nestmate recognition should be studied with explicit reference to the ecological context within which the recognition behavior is expressed (Stuart and Herbers 2000). When colonies are composed of thousands of individuals and are polydomous, the ability to recognize colonial relatives must transcend the confines of the nest itself or include volatile cues in the nest atmosphere, providing an additional signal for nest pertinence. Our results demonstrate for the first time the involvement of nest volatiles in nestmate recognition in ants, in addition to the well-studied cuticular HCs.

Acknowledgements This research was supported by The Israel Binational Science Foundation (Grant no. 1999089 to A.H. and R.V.M.). We thank N. Paz for editorial assistance. The experiments comply with the current laws of Israel.

\section{References}

Boulay R, Quagebeur M, Godzinska E, Lenoir A (1999) Social isolation in ants: evidence of its impact on survivorship and behaviour in Camponotus fellah (Hymenoptera, Formicidae). Sociobiology 33:111-124

Boulay R, Katzav-Gozansky T, Vander Meer R, Hefetz A (2003a) Queen effect on worker social motivation and merging of queenless alien colonies in ants. Proc R Soc Lond B 270:971977

Boulay R, Katzav-Gozansky T, Hefetz A, Lenoir A (2003b) Odour convergence and tolerance between nestmates through trophallaxis and grooming in the ant Camponotus fellah. Insectes Soc 51

Gamboa GJ, Reeve HK, Ferguson ID, Wacker TL (1986) Nestmate recognition in social wasps: the origin and acquisition of recognition odours. Anim Behav 34:685-695

Errard C, Hefetz A (1997) Label familiarity and discriminatory ability of ants reared in mixed groups. Insectes Soc 44:189-198

Jaffe K, Marcuse M (1983) Nestmate recognition and territorial behaviour in the ant Odontomachus bauri Emery (Formicidae: Ponerinae). Insectes Soc 30:466-481 
Kukuk PF, Breed MD, Sobti A, Bell WJ (1977) The contributions of kinship and conditioning to nest recognition and colony member recognition in a primitively eusocial bee, Lasioglossum zephyrum (Hymenopter: Halictidae). Behav Ecol Sociobiol 2:319-327

Lahav S, Soroker V, Hefetz A, Vander Meer RK (1999) Direct behavioral evidence for hydrocarbons as ant recognition discriminators. Naturwissenschaften 86:246-249

Lahav S, Soroker V, Vander Meer RK, Hefetz A (2001) Segregation of colony odor in the desert ant Cataglyphis Niger. J Chem Ecol 27:927-943

Liang D, Silverman J (2000) "You are what you eat": diet modifies cuticular hydrocarbons and nestmate recognition in the Argentine ant, Linepithema humile. Naturwissenschaften 87:412-416

Mann CA, Breed MD (1997) Olfaction in guard honey bee responses to non-nestmates. Ann Entomol Soc Am 90:844-847

Meskali M, Bonavita-Cougourdan A, Provost E, Bagneres AG, Dusticier G, Clément JL (1995) Mechanism underlying cuticular hydrocarbon homogeneity in the ant Camponotus vagus (Scop.) (Hymenopetra: Formicidae): role of postpharyngea glands. J Chem Ecol 21:1127-1148
Soroker V, Vienne C, Hefetz A, Nowbahari E (1994) The postpharyngeal gland as a 'gestalt' organ for nestmate recognition in the ant Cataglyphis niger. Naturwissenschaften 81:510-513

Stuart RJ, Herbers JM (2000) Nest mate recognition in ants with complex colonies: within- and between-population variation. Behav Ecol 11:676-685

Roulston TH, Buczkowski G, Silverman J (2003) Nestmate discrimination in ants: effect of bioassay on aggressive behavior. Insectes Soc 50:151-159

Thomas ML, Parry LJ, Allan RA, Elgar MA (1999) Geographic affinity, cuticular hydrocarbons and colony recognition in the Australian meat ant Iridomyrmex purpureus. Naturwissenschaften 86:87-92

Vander Meer RK, Morel L (1998) Nestmate recognition in ants. In: Vander Meer R, Breed M, Winston M, Espelie K (eds) Pheromone communication in social insects: ants, wasps, bees and termites. Boulder, Colo., Westview, pp 79-103

Wagner D, Tissot M, Cuevas W, Gordon DM (2000) Harvester ants utilize cuticular hydrocarbons in nestmate recognition. J Chem Ecol 26:2245-2257 